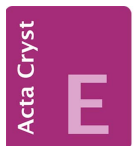

CRYSTALLOGRAPHIC COMMUNICATIONS

ISSN 2056-9890

Received 15 December 2014

Accepted 13 January 2015

Edited by R. F. Baggio, Comisión Nacional de Energía Atómica, Argentina

Keywords: crystal structure; quinoline; benserazide; therapeutic compounds; Parkinson's disease

CCDC reference: 1034614

Supporting information: this article has supporting information at journals.iucr.org/e

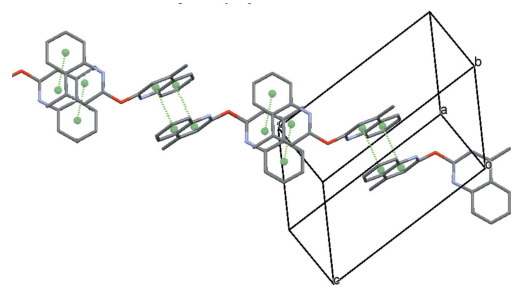

OPEN $\odot$ ACCESS

\section{Crystal structure of 2,2'-oxybis(4-methylquinoline)}

\author{
Anaelle Tilborg
}

Department of Chemistry, University of Namur, 61, Rue de Bruxelles, B-5000 Namur, Belgium. *Correspondence e-mail: anaelle.tilborg@unamur.be

The title compound, $\mathrm{C}_{20} \mathrm{H}_{16} \mathrm{~N}_{2} \mathrm{O}$, (I), has been unwittingly obtained from the slow evaporation of a saturated solution of commercial benserazide hydrochloride [benserazide, (II), being one of the principal therapeutic compounds used for the management of Parkinson's disease, mostly used in combination with levodopa]. The molecule of (I) is composed of two planar 4-methylquinoline aromatic moieties [maximum deviations of $0.0104(18)$ and 0.016 (2) $\AA$ ] , almost perpendicular to each other [dihedral angle $=89.5(2)^{\circ}$ ], bridged by an $\mathrm{O}$ atom. The supramolecular organization consists of a $\pi$-bonded chain, resulting from the stacking of molecules related by inversion centres located along direction [111].

\section{Chemical context}

Parkinson's disease is a degenerative disorder of the central nervous system, resulting from the death of dopaminegenerating cells, mostly located in the mid-brain. The most obvious symptoms are movement-related: uncontrolled shaking, rigidity, slowness of movement and difficulty in walking. However, behavioral problems and psychiatric depression may also arise (Samii et al., 2004). Symptomatic treatment of Parkinson's disease includes daily dopamine administration, principally through L-DOPA (or levodopa) or carbidopa (both being precursors of dopamine) brain metabolization.<smiles>Cc1cc(Oc2cc(C)c3ccccc3n2)nc2ccccc12</smiles>

(I)<smiles>NC(CO)C(=O)NNCc1ccc(O)c(O)c1O</smiles>

(II)

Benserazide [also called Serazide or Ro-4-4602, (II) in the Scheme] is an aromatic L-amino acid decarboxylase inhibitor and a DOPA decarboxylase inhibitor unable to cross the blood-brain barrier. It is used in combination with levodopa for the symptomatic management of Parkinson's disease (Clark et al., 1973; Campanella \& Pennetta, 1974; Bortolanza et al., 2015). 


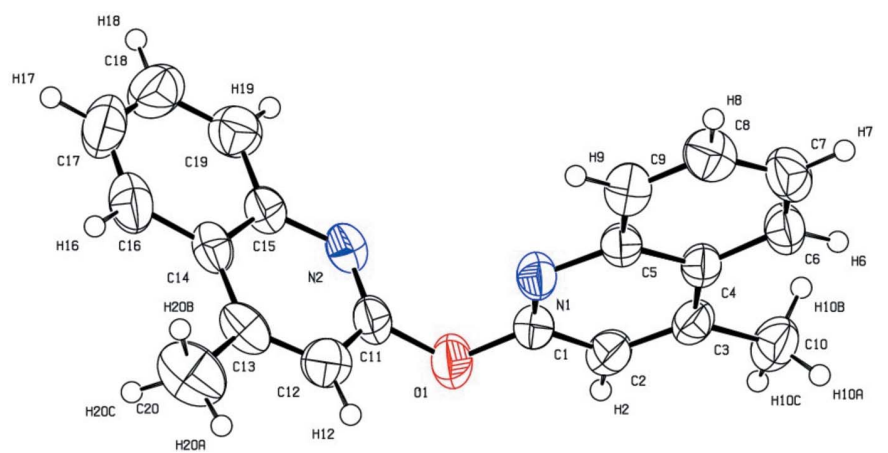

Figure 1

The molecular structure and atom numbering of the title compound. Displacement ellipsoids for the non- $\mathrm{H}$ atoms are drawn at the $50 \%$ probability level.

As benserazide is always administered in combination therapy, it appeared to be a good candidate to search for a solid-state crystalline phase involving it with another therapeutic molecule, also active in the treatment of Parkinson's disease. However, little information could be retrieved on the structural aspects of benserazide and, as a first step, recrystallization attempts of the molecule alone have been launched. These crystallization assays have been so far fruitless, but resulted instead in the unwitting obtention of a new molecule, 2,2'-oxybis(4-methylquinoline) (I) with formula $\mathrm{C}_{20} \mathrm{H}_{16} \mathrm{ON}_{2}$, which is reported herein.

\section{Structural commentary}

The geometry of (I) is fairly predictable, with all bond lengths and valence angles being in the expected range for organic compounds (Allen et al., 1987). The molecule consists of two planar 4-methylquinoline aromatic moieties [the maximum deviations from the mean plane are 0.0104 (18) $\AA$ for $\mathrm{C} 1$ in the

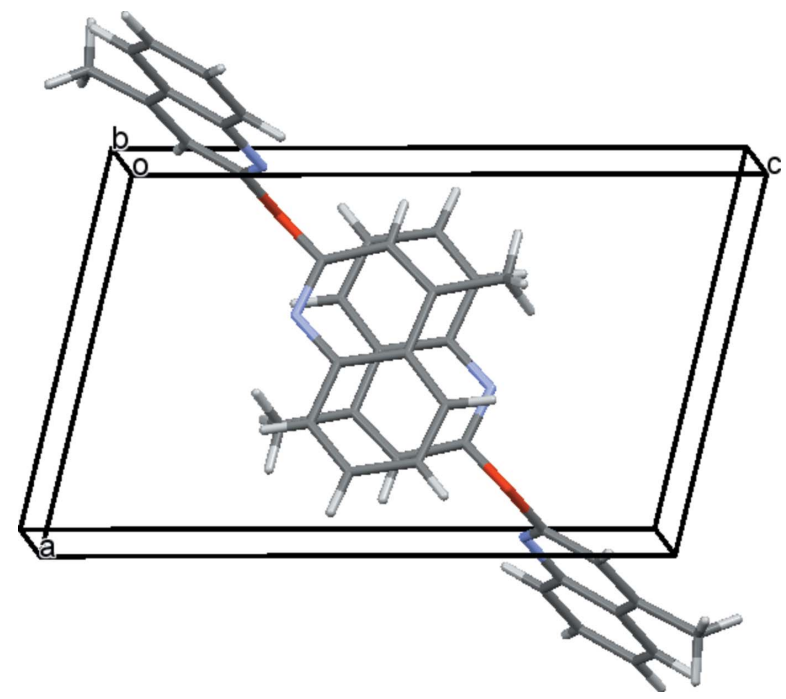

Figure 2

Packing diagram showing one of the $\pi-\pi$ interactions, stacked around $\left(\frac{1}{2}, \frac{1}{2}, \frac{1}{2}\right)$.
Table 1

$\pi-\pi$ stacking interactions $\left(\AA,^{\circ}\right)$.

$C g 1, C g 2, C g 3$ and $C g 4$ are the centroids of the N1/C1-C5, N2/C11-C15, C4-C9 and $\mathrm{C} 14-\mathrm{C} 19$ rings, respectively. $\mathrm{Cg} \cdots \mathrm{Cg}$ is the intercentroid distance, the dihedral angle is between the ring planes and mpd is the mean perpendicular distance between a centroid and the opposite plane.

\begin{tabular}{llll}
\hline & $C g \cdots C g$ & dihedral angle & mpd \\
\hline$C g 1 \cdots C g 1^{\mathrm{i}}$ & $3.7849(11)$ & 0 & $3.4446(7)$ \\
$C g 1 \cdots C g 3^{\mathrm{i}}$ & $3.7775(11)$ & $0.83(8)$ & $3.4345(10)$ \\
$C g 2 \cdots C g 2^{\text {ii }}$ & $3.6036(11)$ & 0 & $3.4395(7)$ \\
$C g 2 \cdots C g 4^{\text {ii }}$ & $3.8817(12)$ & $0.73(10)$ & $3.4462(19)$ \\
\hline
\end{tabular}

Symmetry codes: (i) $-x,-y,-z$; (ii) $1-x, 1-y, 1-z$.

N1,C1-C9 moiety and 0.016 (2) $\AA$ for C13 in the N2,C11-C19 unit], almost perpendicular to each other [dihedral angle = $\left.89.5(2)^{\circ}\right]$ and bound by an oxygen atom which forms an ether link (Fig. 1).

\section{Supramolecular features}

The crystal packing organization is essentially the result of two different types of $\pi$-stacking interactions involving inversionrelated molecules. Table 1 gives a survey of these $\pi-\pi$ stacking interactions, in one case around $\left(\frac{1}{2}, \frac{1}{2}, \frac{1}{2}\right)$ (Fig. 2) and in the other case around $(0,0,0) ;(1,1,1)$ (Fig. 3$)$. The overall effect of these interactions is the formation of chains parallel to [111] (Fig. 4). As expected from the lack of efficient hydrogen-bond donors, no significant hydrogen bonds linking the chains are present in the structure, as a result of which their mutual interaction is rather weak.

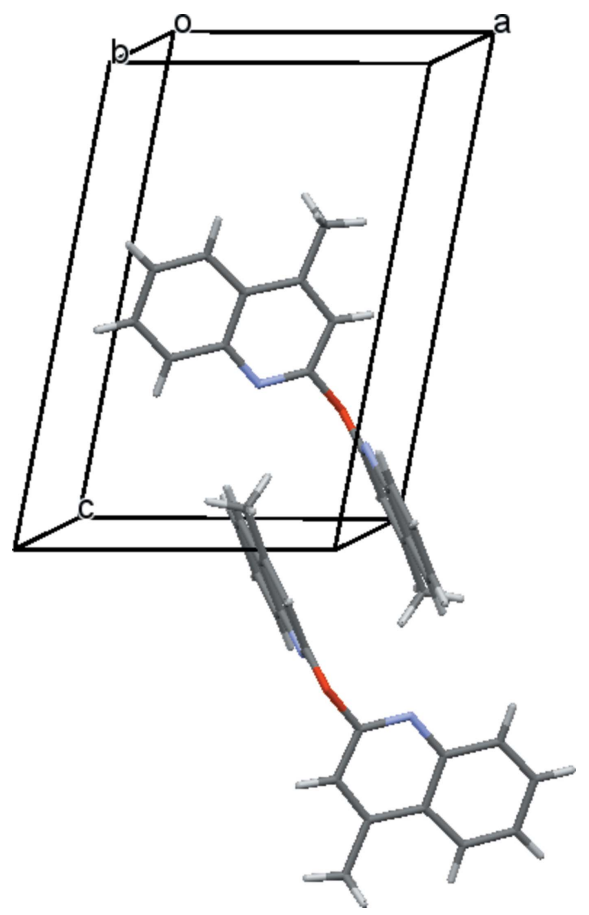

Figure 3

Packing diagram showing the second type of $\pi-\pi$ interaction, stacked around $(1,1,1)$. 


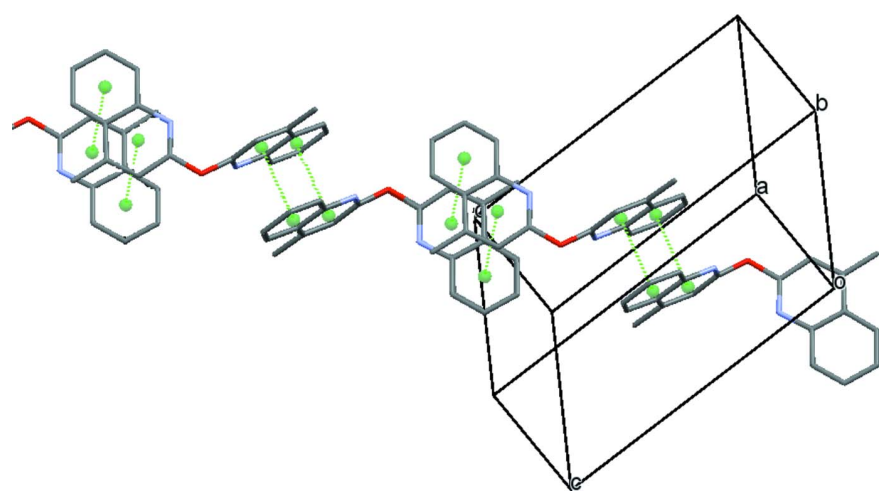

Figure 4

The [111] chain resulting from the two types of $\pi-\pi$ interactions.

\section{Database survey}

A systematic research in the Cambridge Structural Database (CSD; Version 5.35, update November 2014; Groom \& Allen, 2014) using ConQuest (Bruno et al., (2002) revealed some structures fairly similar to (I), which are presented in Fig. 5 and identified by their CSD refcodes: MOSLAI (Hassan et al., 2009) and JUBRAZ (Liu et al., 1992), the main difference residing in the number and relative position of the nitrogen atoms in the aromatic rings.

\section{Synthesis and crystallization}

Prismatic colourless crystals of 2,2'-oxybis(4-methylquinoline) were grown from a $2 \mathrm{ml}$ aqueous saturated solution of benserazide hydrochloride (purchased from Sigma-Aldrich, Steinheim, Germany; purity level claimed > 98\%) (9.3 mg) that was allowed to evaporate slowly at room temperature over 7 days.

Several trials of slow evaporation of aqueous solutions under different temperature conditions (from 277 to $313 \mathrm{~K}$ ) provided in all cases the same crystals, with the same unit-cell parameters. The main assumption is that the benserazide hydrochloride has undergone a fundamental structure transformation during the aqueous recrystallization assays, but work is in progress to understand the mechanism, which does<smiles>CO[Mg]</smiles><smiles></smiles>

Figure 5

Two similar structures in the CSD [refcodes MOSLAI (Hassan et al., 2009) and JUBRAZ (Liu et al., 1992)].
Table 2

Experimental details.

\begin{tabular}{|c|c|}
\hline \multicolumn{2}{|l|}{ Crystal data } \\
\hline Chemical formula & $\mathrm{C}_{20} \mathrm{H}_{16} \mathrm{~N}_{2} \mathrm{O}$ \\
\hline$M_{\mathrm{r}}$ & 300.35 \\
\hline Crystal system, space group & Triclinic, $P \overline{1}$ \\
\hline Temperature (K) & 293 \\
\hline$a, b, c(\AA)$ & $7.8858(5), 7.9226(8), 13.0182(13)$ \\
\hline$\alpha, \beta, \gamma\left({ }^{\circ}\right)$ & $104.267(9), 103.576(7), 91.967(7)$ \\
\hline$V\left(\AA^{3}\right)$ & $762.54(13)$ \\
\hline$Z$ & 2 \\
\hline Radiation type & Мо $K \alpha$ \\
\hline$\mu\left(\mathrm{mm}^{-1}\right)$ & 0.08 \\
\hline Crystal size $(\mathrm{mm})$ & $0.5 \times 0.35 \times 0.25$ \\
\hline \multicolumn{2}{|l|}{ Data collection } \\
\hline Diffractometer & $\begin{array}{l}\text { Oxford Diffraction Xcalibur } \\
\text { (Ruby, Gemini) ultra }\end{array}$ \\
\hline Absorption correction & $\begin{array}{l}\text { Multi-scan (CrysAlis PRO; Oxford } \\
\text { Diffraction, 2006) }\end{array}$ \\
\hline$T_{\min }, T_{\max }$ & $0.960,1.000$ \\
\hline $\begin{array}{l}\text { No. of measured, independent and } \\
\text { observed }[I>2 \sigma(I)] \text { reflections }\end{array}$ & $3234,2207,1707$ \\
\hline$R_{\text {int }}$ & 0.024 \\
\hline$(\sin \theta / \lambda)_{\max }\left(\AA^{-1}\right)$ & 0.595 \\
\hline \multicolumn{2}{|l|}{ Refinement } \\
\hline$R\left[F^{2}>2 \sigma\left(F^{2}\right)\right], w R\left(F^{2}\right), S$ & $0.046,0.133,1.03$ \\
\hline No. of reflections & 2256 \\
\hline No. of parameters & 232 \\
\hline No. of restraints & 6 \\
\hline $\mathrm{H}$-atom treatment & $\begin{array}{l}\mathrm{H} \text { atoms treated by a mixture of } \\
\text { independent and constrained } \\
\text { refinement }\end{array}$ \\
\hline$\Delta \rho_{\max }, \Delta \rho_{\min }\left(\mathrm{e} \AA^{-3}\right)$ & $0.18,-0.14$ \\
\hline
\end{tabular}

Computer programs: CrysAlis PRO (Oxford Diffraction, 2006), SIR92 (Altomare et al., 1994), SHELXL97 (Sheldrick, 2015), ORTEPIII (Burnett \& Johnson, 1996), PLATON (Spek, 2009) and Mercury (Macrae et al., 2006).

not seem to be obvious. Compound (I) could also be a byproduct coming from a earlier step in the benserazide synthesis process (even if the quantity of crystalline material retrieved is relatively important). A calorimetric study has been undertaken on the crystalline material, and differential scanning calorimetry (DSC) provides an onset temperature (considered as the melting point) of $419.3 \mathrm{~K}$, with no significant endo- or exothermic event before the fusion point. No spontaneous recrystallization occurs when the melt is allowed to cool down.

\section{Refinement}

Crystal data, data collection and structure refinement details are summarized in Table 2. The methyl $\mathrm{H}$ atoms were located from difference Fourier maps and their positions refined freely. All other $\mathrm{H}$ atoms were placed at idealized positions and allowed to ride on their parent atoms, with $\mathrm{C}-\mathrm{H}$ distances of $0.93 \AA$ and with $U_{\text {iso }}(\mathrm{H})=1.2 U_{\text {eq }}(\mathrm{C})$.

\section{Acknowledgements}

This work was supported by the Fonds National de la Recherche Scientifique (FRS-FNRS, Belgium). AT acknowledges Professor J. Wouters and B. Norberg for their fruitful work and input to the project. 


\section{References}

Allen, F. H., Kennard, O., Watson, D. G., Brammer, L., Orpen, A. G. \& Taylor, R. (1987). J. Chem. Soc. Perkin Trans. 2, pp. S1-19.

Altomare, A., Cascarano, G., Giacovazzo, C., Guagliardi, A., Burla, M. C., Polidori, G. \& Camalli, M. (1994). J. Appl. Cryst. 27, 435.

Bortolanza, M., Cavalcanti-Kiwiatkoski, R., Padovan-Neto, F. E., Da Silva, C. A., Mitkovski, M., Raisman-Vozari, R. \& Del-Bel, E. (2015). Neurobiol. Dis. 73, 377-387.

Bruno, I. J., Cole, J. C., Edgington, P. R., Kessler, M., Macrae, C. F., McCabe, P., Pearson, J. \& Taylor, R. (2002). Acta Cryst. B58, 389397.

Burnett, M. N. \& Johnson, C. K. (1996). ORTEPIII. Report ORNL6895. Oak Ridge National Laboratory, Tennessee, USA.

Campanella, G. \& Pennetta, R. (1974). Acta Neurol. 29, 252-263.
Clark, W. G., Oldendorf, W. H. \& Dewhurst, W. G. (1973). J. Pharm. Pharmacol. 25, 416-418.

Groom, C. R. \& Allen, F. H. (2014). Angew. Chem. Int. Ed. 53, 662671.

Hassan, N. D., Tajuddin, H. A., Abdullah, Z. \& Ng, S. W. (2009). Acta Cryst. E65, o732.

Liu, H., Wang, X. \& Zhang, X. (1992). Acta Cryst. C48, 2096-2098.

Macrae, C. F., Edgington, P. R., McCabe, P., Pidcock, E., Shields, G. P., Taylor, R., Towler, M. \& van de Streek, J. (2006). J. Appl. Cryst. 39, 453-457.

Oxford Diffraction (2006). CrysAlis PRO. Oxford Diffraction Ltd, Abingdon, England.

Samii, A., Nutt, J. G. \& Ransom, B. R. (2004). Lancet, 363, 1783-1793.

Sheldrick, G. M. (2015). Acta Cryst. C71, 3-8.

Spek, A. L. (2009). Acta Cryst. D65, 148-155. 


\section{supporting information}

Acta Cryst. (2015). E71, 180-183 [doi:10.1107/S2056989015000717]

\section{Crystal structure of 2,2'-oxybis(4-methylquinoline)}

\section{Anaelle Tilborg}

\section{Computing details}

Data collection: CrysAlis PRO (Oxford Diffraction, 2006); cell refinement: CrysAlis PRO (Oxford Diffraction, 2006); data reduction: CrysAlis PRO (Oxford Diffraction, 2006); program(s) used to solve structure: SIR92 (Altomare et al., 1994); program(s) used to refine structure: SHELXL97 (Sheldrick, 2015); molecular graphics: ORTEPIII (Burnett \& Johnson, 1996), PLATON (Spek, 2009) and Mercury (Macrae et al., 2006); software used to prepare material for publication: SHELXL97 (Sheldrick, 2015).

\section{2,2'-Oxybis(4-methylquinoline)}

\section{Crystal data}

$\mathrm{C}_{20} \mathrm{H}_{16} \mathrm{~N}_{2} \mathrm{O}$

$M_{r}=300.35$

Triclinic, $P \overline{1}$

Hall symbol: -P 1

$a=7.8858(5) \AA$

$b=7.9226(8) \AA$

$c=13.0182(13) \AA$

$\alpha=104.267(9)^{\circ}$

$\beta=103.576(7)^{\circ}$

$\gamma=91.967(7)^{\circ}$

$V=762.54(13) \AA^{3}$

Data collection

Oxford Diffraction Xcalibur (Ruby, Gemini) ultra diffractometer

Radiation source: fine-focus sealed tube Graphite monochromator

Detector resolution: 10.3712 pixels $\mathrm{mm}^{-1}$ $\omega$ scans

Absorption correction: multi-scan

(CrysAlis PRO; Oxford Diffraction, 2006)

Refinement

Refinement on $F^{2}$

Least-squares matrix: full

$R\left[F^{2}>2 \sigma\left(F^{2}\right)\right]=0.046$

$w R\left(F^{2}\right)=0.133$

$S=1.03$

2256 reflections

232 parameters

6 restraints

$$
\begin{aligned}
& Z=2 \\
& F(000)=316 \\
& D_{\mathrm{x}}=1.308 \mathrm{Mg} \mathrm{m}^{-3}
\end{aligned}
$$

Mo $K \alpha$ radiation, $\lambda=0.71073 \AA$

Cell parameters from 1411 reflections

$\theta=3.7-28.7^{\circ}$

$\mu=0.08 \mathrm{~mm}^{-1}$

$T=293 \mathrm{~K}$

Prism, colourless

$0.5 \times 0.35 \times 0.25 \mathrm{~mm}$

$T_{\min }=0.960, T_{\max }=1.000$

3234 measured reflections

2207 independent reflections

1707 reflections with $I>2 \sigma(I)$

$R_{\text {int }}=0.024$

$\theta_{\max }=25.0^{\circ}, \theta_{\min }=3.5^{\circ}$

$h=-7 \rightarrow 9$

$k=-9 \rightarrow 5$

$l=-14 \rightarrow 15$

0 constraints

Primary atom site location: structure-invariant direct methods

Secondary atom site location: difference Fourier map

Hydrogen site location: inferred from neighbouring sites 
$\mathrm{H}$ atoms treated by a mixture of independent and constrained refinement

$w=1 /\left[\sigma^{2}\left(F_{\mathrm{o}}^{2}\right)+(0.0652 P)^{2}+0.0421 P\right]$

where $P=\left(F_{\mathrm{o}}^{2}+2 F_{\mathrm{c}}^{2}\right) / 3$

$$
\begin{aligned}
& (\Delta / \sigma)_{\max }<0.001 \\
& \Delta \rho_{\max }=0.18 \mathrm{e} \AA^{-3} \\
& \Delta \rho_{\min }=-0.14 \mathrm{e} \AA^{-3}
\end{aligned}
$$

\section{Special details}

Experimental. Absorption correction: CrysAlis PRO, Empirical absorption correction using spherical harmonics, implemented in SCALE3 ABSPACK scaling algorithm.

Geometry. All e.s.d.'s (except the e.s.d. in the dihedral angle between two 1.s. planes) are estimated using the full covariance matrix. The cell e.s.d.'s are taken into account individually in the estimation of e.s.d.'s in distances, angles and torsion angles; correlations between e.s.d.'s in cell parameters are only used when they are defined by crystal symmetry. An approximate (isotropic) treatment of cell e.s.d.'s is used for estimating e.s.d.'s involving l.s. planes.

Refinement. Refinement of $F^{2}$ against ALL reflections. The weighted $R$-factor $w R$ and goodness of fit $S$ are based on $F^{2}$, conventional $R$-factors $R$ are based on $F$, with $F$ set to zero for negative $F^{2}$. The threshold expression of $F^{2}>\sigma\left(F^{2}\right)$ is used only for calculating $R$-factors(gt) etc. and is not relevant to the choice of reflections for refinement. $R$-factors based on $F^{2}$

\begin{tabular}{|c|c|c|c|c|}
\hline & $x$ & $y$ & $z$ & $U_{\text {iso }} * / U_{\text {eq }}$ \\
\hline $\mathrm{O} 1$ & $0.13084(17)$ & $0.38253(18)$ & $0.26211(12)$ & $0.0696(5)$ \\
\hline N1 & $-0.01449(17)$ & $0.1056(2)$ & $0.20704(12)$ & $0.0470(4)$ \\
\hline $\mathrm{N} 2$ & 0.38771 (19) & $0.2735(2)$ & $0.32211(12)$ & $0.0530(4)$ \\
\hline $\mathrm{C} 1$ & $0.0147(2)$ & $0.2537(2)$ & $0.18566(15)$ & $0.0482(5)$ \\
\hline $\mathrm{C} 2$ & $-0.0638(2)$ & $0.2993(3)$ & $0.08862(16)$ & $0.0507(5)$ \\
\hline $\mathrm{C} 3$ & $-0.1801(2)$ & $0.1811(3)$ & $0.00729(15)$ & $0.0458(5)$ \\
\hline $\mathrm{C} 4$ & $-0.2184(2)$ & $0.0150(2)$ & $0.02535(13)$ & $0.0415(4)$ \\
\hline $\mathrm{C} 5$ & $-0.1336(2)$ & $-0.0165(2)$ & $0.12652(14)$ & $0.0413(4)$ \\
\hline C6 & $-0.3353(2)$ & -0.1194 (3) & $-0.05297(16)$ & $0.0535(5)$ \\
\hline $\mathrm{C} 7$ & $-0.3664(2)$ & $-0.2761(3)$ & $-0.03220(18)$ & $0.0626(6)$ \\
\hline $\mathrm{C} 8$ & $-0.2839(2)$ & -0.3060 & $0.06810(18)$ & $0.0599(6)$ \\
\hline C9 & $-0.1692(2)$ & $-0.1786(3)$ & $0.14565(16)$ & $0.0528(5)$ \\
\hline $\mathrm{C} 10$ & $-0.2614(3)$ & $0.2203(4)$ & -0.09923 (19) & $0.0620(6)$ \\
\hline $\mathrm{C} 11$ & $0.2483(2)$ & $0.3332(2)$ & $0.34593(15)$ & $0.0506(5)$ \\
\hline C12 & $0.2112(2)$ & $0.3641(3)$ & $0.44746(18)$ & $0.0560(5)$ \\
\hline $\mathrm{C} 13$ & 0.3299 & $0.3308(2)$ & $0.53294(16)$ & $0.0535(5)$ \\
\hline $\mathrm{C} 14$ & $0.4866(2)$ & $0.2617(2)$ & $0.51214(15)$ & $0.0479(5)$ \\
\hline $\mathrm{C} 15$ & $0.5100(2)$ & $0.2357(2)$ & $0.40549(15)$ & $0.0472(5)$ \\
\hline $\mathrm{C} 16$ & $0.6200(3)$ & $0.2177(3)$ & $0.59128(18)$ & $0.0655(6)$ \\
\hline $\mathrm{C} 17$ & $0.7657(3)$ & 0.1528 & $0.5655(2)$ & $0.0808(8)$ \\
\hline C18 & $0.7892(3)$ & 0.1314 & $0.4606(2)$ & $0.0797(7)$ \\
\hline C19 & 0.6640 & $0.1713(3)$ & $0.38176(19)$ & $0.0644(6)$ \\
\hline $\mathrm{C} 20$ & $0.2972(4)$ & $0.3663(4)$ & $0.6447(2)$ & $0.0863(8)$ \\
\hline $\mathrm{H} 2$ & -0.0360 & 0.4095 & 0.0804 & $0.061 *$ \\
\hline H6 & -0.3920 & -0.1009 & -0.1199 & $0.064 *$ \\
\hline $\mathrm{H} 7$ & -0.4432 & -0.3639 & -0.0852 & $0.075^{*}$ \\
\hline $\mathrm{H} 8$ & -0.3071 & -0.4128 & 0.0821 & $0.072 *$ \\
\hline H9 & -0.1140 & -0.1998 & 0.2120 & $0.063 *$ \\
\hline $\mathrm{H} 10 \mathrm{~A}$ & -0.386 & $0.211(3)$ & $-0.1129(17)$ & $0.070(6)^{*}$ \\
\hline H10B & $-0.229(3)$ & 0.137 (3) & $-0.159(2)$ & $0.081(7)^{*}$ \\
\hline
\end{tabular}
are statistically about twice as large as those based on $F$, and $R$-factors based on ALL data will be even larger.

Fractional atomic coordinates and isotropic or equivalent isotropic displacement parameters $\left(\AA^{2}\right)$ 
supporting information

\begin{tabular}{lllll} 
H10C & $-0.219(3)$ & $0.337(4)$ & $-0.100(2)$ & $0.096(8)^{*}$ \\
H12 & 0.1062 & 0.4072 & 0.4572 & $0.067^{*}$ \\
H16 & 0.6078 & 0.2334 & 0.6623 & $0.079^{*}$ \\
H17 & 0.8511 & 0.1222 & 0.6186 & $0.097^{*}$ \\
H18 & 0.8912 & 0.0895 & 0.4446 & $0.096^{*}$ \\
H19 & 0.6801 & 0.1560 & 0.3116 & $0.077^{*}$ \\
H20A & $0.186(2)$ & $0.408(3)$ & $0.648(3)$ & $0.137(12)^{*}$ \\
H20B & $0.306(3)$ & $0.259(3)$ & $0.669(3)$ & $0.140(12)^{*}$ \\
H20C & $0.389(3)$ & $0.451(3)$ & $0.698(2)$ & $0.129(11)^{*}$ \\
\hline
\end{tabular}

Atomic displacement parameters $\left(\AA^{2}\right)$

\begin{tabular}{lllllll}
\hline & $U^{11}$ & $U^{22}$ & $U^{33}$ & $U^{12}$ & $U^{13}$ & $U^{23}$ \\
\hline O1 & $0.0706(9)$ & $0.0484(9)$ & $0.0657(10)$ & $-0.0002(6)$ & $-0.0204(7)$ & $0.0072(7)$ \\
N1 & $0.0449(9)$ & $0.0537(10)$ & $0.0366(9)$ & $0.0033(7)$ & $0.0035(6)$ & $0.0079(8)$ \\
N2 & $0.0590(11)$ & $0.0537(10)$ & $0.0368(9)$ & $0.0031(8)$ & $0.0042(7)$ & $0.0023(8)$ \\
C1 & $0.0407(10)$ & $0.0487(11)$ & $0.0465(12)$ & $0.0059(8)$ & $0.0027(8)$ & $0.0042(10)$ \\
C2 & $0.0466(11)$ & $0.0502(11)$ & $0.0542(12)$ & $0.0094(8)$ & $0.0066(9)$ & $0.0165(10)$ \\
C3 & $0.0375(10)$ & $0.0618(12)$ & $0.0397(10)$ & $0.0139(8)$ & $0.0099(7)$ & $0.0146(10)$ \\
C4 & $0.0333(10)$ & $0.0552(11)$ & $0.0349(10)$ & $0.0071(7)$ & $0.0105(7)$ & $0.0072(9)$ \\
C5 & $0.0361(10)$ & $0.0496(11)$ & $0.0371(10)$ & $0.0041(7)$ & $0.0116(7)$ & $0.0072(9)$ \\
C6 & $0.0456(11)$ & $0.0677(14)$ & $0.0384(11)$ & $0.0031(9)$ & $0.0054(8)$ & $0.0030(10)$ \\
C7 & $0.0480(12)$ & $0.0649(14)$ & $0.0609(14)$ & $-0.0080(9)$ & $0.0089(9)$ & $-0.0032(12)$ \\
C8 & $0.0537(13)$ & $0.0568(13)$ & $0.0692(15)$ & $-0.0040(9)$ & $0.0203(10)$ & $0.0128(12)$ \\
C9 & $0.0479(11)$ & $0.0644(13)$ & $0.0487(12)$ & $0.0016(9)$ & $0.0140(8)$ & $0.0182(11)$ \\
C10 & $0.0562(15)$ & $0.0813(18)$ & $0.0507(14)$ & $0.0172(12)$ & $0.0076(10)$ & $0.0254(13)$ \\
C11 & $0.0506(12)$ & $0.0446(11)$ & $0.0432(12)$ & $-0.0029(8)$ & $-0.0034(9)$ & $0.0026(9)$ \\
C12 & $0.0456(11)$ & $0.0517(12)$ & $0.0681(14)$ & $-0.0010(8)$ & $0.0162(9)$ & $0.0099(11)$ \\
C13 & $0.0682(13)$ & $0.0445(11)$ & $0.0459(12)$ & $-0.0100(9)$ & $0.0213(9)$ & $0.0035(9)$ \\
C14 & $0.0535(12)$ & $0.0425(11)$ & $0.0406(11)$ & $-0.0094(8)$ & $0.0038(8)$ & $0.0074(9)$ \\
C15 & $0.0476(11)$ & $0.0448(11)$ & $0.0422(11)$ & $-0.0021(8)$ & $0.0064(8)$ & $0.0042(9)$ \\
C16 & $0.0767(15)$ & $0.0604(13)$ & $0.0480(13)$ & $-0.0077(11)$ & $-0.0051(10)$ & $0.0151(11)$ \\
C17 & $0.0659(16)$ & $0.0710(16)$ & $0.086(2)$ & $-0.0015(12)$ & $-0.0221(13)$ & $0.0247(15)$ \\
C18 & $0.0536(14)$ & $0.0674(16)$ & $0.108(2)$ & $0.0078(10)$ & $0.0065(13)$ & $0.0165(15)$ \\
C19 & $0.0631(14)$ & $0.0631(14)$ & $0.0649(14)$ & $0.0069(10)$ & $0.0185(10)$ & $0.0107(11)$ \\
C20 & $0.118(2)$ & $0.087(2)$ & $0.0657(18)$ & $0.0003(17)$ & $0.0483(16)$ & $0.0180(16)$ \\
& & & & & & \\
\hline
\end{tabular}

Geometric parameters $\left(\AA,{ }^{\circ}\right)$

\begin{tabular}{llll}
\hline $\mathrm{O} 1-\mathrm{C} 1$ & $1.371(2)$ & $\mathrm{C} 10-\mathrm{H} 10 \mathrm{C}$ & $0.97(3)$ \\
$\mathrm{O} 1-\mathrm{C} 11$ & $1.401(2)$ & $\mathrm{C} 10-\mathrm{H} 10 \mathrm{~A}$ & $0.95(2)$ \\
$\mathrm{N} 1-\mathrm{C} 1$ & $1.296(2)$ & $\mathrm{C} 10-\mathrm{H} 10 \mathrm{~B}$ & $0.97(3)$ \\
$\mathrm{N} 1-\mathrm{C} 5$ & $1.376(2)$ & $\mathrm{C} 11-\mathrm{C} 12$ & $1.386(3)$ \\
$\mathrm{N} 2-\mathrm{C} 11$ & $1.285(2)$ & $\mathrm{C} 12-\mathrm{H} 12$ & 0.9300 \\
$\mathrm{~N} 2-\mathrm{C} 15$ & $1.373(2)$ & $\mathrm{C} 13-\mathrm{C} 12$ & $1.363(3)$ \\
$\mathrm{C} 2-\mathrm{C} 3$ & $\mathrm{C} 13-\mathrm{C} 20$ & $1.497(3)$ \\
$\mathrm{C} 2-\mathrm{C} 1$ & $1.355(3)$ & $\mathrm{C} 14-\mathrm{C} 16$ & $1.408(3)$ \\
$\mathrm{C} 2-\mathrm{H} 2$ & $1.408(2)$ & $\mathrm{C} 14-\mathrm{C} 15$ & $1.409(3)$
\end{tabular}




\begin{tabular}{|c|c|c|c|}
\hline $\mathrm{C} 3-\mathrm{C} 10$ & $1.496(3)$ & $\mathrm{C} 14-\mathrm{C} 13$ & $1.426(3)$ \\
\hline $\mathrm{C} 4-\mathrm{C} 6$ & $1.407(3)$ & $\mathrm{C} 15-\mathrm{C} 19$ & $1.404(3)$ \\
\hline $\mathrm{C} 4-\mathrm{C} 3$ & $1.427(3)$ & $\mathrm{C} 16-\mathrm{C} 17$ & $1.354(3)$ \\
\hline $\mathrm{C} 5-\mathrm{C} 9$ & $1.398(2)$ & $\mathrm{C} 16-\mathrm{H} 16$ & 0.9300 \\
\hline $\mathrm{C} 5-\mathrm{C} 4$ & $1.416(2)$ & $\mathrm{C} 17-\mathrm{H} 17$ & 0.9300 \\
\hline $\mathrm{C} 6-\mathrm{C} 7$ & $1.360(3)$ & $\mathrm{C} 18-\mathrm{C} 17$ & $1.391(4)$ \\
\hline $\mathrm{C} 6-\mathrm{H} 6$ & 0.9300 & $\mathrm{C} 18-\mathrm{H} 18$ & 0.9300 \\
\hline $\mathrm{C} 7-\mathrm{H} 7$ & 0.9300 & $\mathrm{C} 19-\mathrm{C} 18$ & $1.354(3)$ \\
\hline $\mathrm{C} 8-\mathrm{C} 7$ & $1.396(3)$ & $\mathrm{C} 19-\mathrm{H} 19$ & 0.9300 \\
\hline $\mathrm{C} 8-\mathrm{H} 8$ & 0.9300 & $\mathrm{C} 20-\mathrm{H} 20 \mathrm{~A}$ & $0.956(18)$ \\
\hline $\mathrm{C} 9-\mathrm{C} 8$ & $1.365(3)$ & $\mathrm{C} 20-\mathrm{H} 20 \mathrm{~B}$ & $0.981(18)$ \\
\hline $\mathrm{C} 9-\mathrm{H} 9$ & 0.9300 & $\mathrm{C} 20-\mathrm{H} 20 \mathrm{C}$ & $0.978(19)$ \\
\hline $\mathrm{O} 1-\mathrm{C} 1-\mathrm{C} 2$ & $114.47(16)$ & $\mathrm{C} 9-\mathrm{C} 8-\mathrm{H} 8$ & 119.9 \\
\hline $\mathrm{N} 1-\mathrm{C} 1-\mathrm{O} 1$ & $119.50(16)$ & $\mathrm{C} 11-\mathrm{N} 2-\mathrm{C} 15$ & $116.43(16)$ \\
\hline $\mathrm{N} 1-\mathrm{C} 1-\mathrm{C} 2$ & $126.03(17)$ & $\mathrm{C} 11-\mathrm{C} 12-\mathrm{H} 12$ & 120.3 \\
\hline $\mathrm{N} 1-\mathrm{C} 5-\mathrm{C} 9$ & $118.13(16)$ & $\mathrm{C} 12-\mathrm{C} 11-\mathrm{O} 1$ & 118.03 \\
\hline $\mathrm{N} 1-\mathrm{C} 5-\mathrm{C} 4$ & $122.58(15)$ & $\mathrm{C} 12-\mathrm{C} 13-\mathrm{C} 14$ & $117.55(18)$ \\
\hline $\mathrm{N} 2-\mathrm{C} 11-\mathrm{C} 12$ & $126.19(17)$ & $\mathrm{C} 12-\mathrm{C} 13-\mathrm{C} 20$ & $121.2(2)$ \\
\hline $\mathrm{N} 2-\mathrm{C} 11-\mathrm{O} 1$ & $115.61(18)$ & $\mathrm{C} 13-\mathrm{C} 12-\mathrm{C} 11$ & $119.38(18)$ \\
\hline $\mathrm{N} 2-\mathrm{C} 15-\mathrm{C} 19$ & $117.56(18)$ & $\mathrm{C} 13-\mathrm{C} 12-\mathrm{H} 12$ & 120.3 \\
\hline $\mathrm{N} 2-\mathrm{C} 15-\mathrm{C} 14$ & $122.48(17)$ & $\mathrm{C} 13-\mathrm{C} 20-\mathrm{H} 20 \mathrm{~A}$ & $113.8(19)$ \\
\hline $\mathrm{C} 1-\mathrm{O} 1-\mathrm{C} 11$ & $117.49(14)$ & $\mathrm{C} 13-\mathrm{C} 20-\mathrm{H} 20 \mathrm{~B}$ & $109(2)$ \\
\hline $\mathrm{C} 1-\mathrm{N} 1-\mathrm{C} 5$ & $116.00(15)$ & $\mathrm{C} 13-\mathrm{C} 20-\mathrm{H} 20 \mathrm{C}$ & $111.1(18)$ \\
\hline $\mathrm{C} 1-\mathrm{C} 2-\mathrm{H} 2$ & 120.3 & $\mathrm{C} 14-\mathrm{C} 13-\mathrm{C} 20$ & $121.2(2)$ \\
\hline $\mathrm{C} 2-\mathrm{C} 3-\mathrm{C} 4$ & $117.60(16)$ & $\mathrm{C} 14-\mathrm{C} 16-\mathrm{H} 16$ & 119.5 \\
\hline $\mathrm{C} 2-\mathrm{C} 3-\mathrm{C} 10$ & $121.44(19)$ & $\mathrm{C} 15-\mathrm{C} 14-\mathrm{C} 13$ & $117.96(16)$ \\
\hline $\mathrm{C} 3-\mathrm{C} 2-\mathrm{C} 1$ & $119.35(17)$ & $\mathrm{C} 15-\mathrm{C} 19-\mathrm{H} 19$ & 119.9 \\
\hline $\mathrm{C} 3-\mathrm{C} 2-\mathrm{H} 2$ & 120.3 & $\mathrm{C} 16-\mathrm{C} 14-\mathrm{C} 15$ & $117.77(19)$ \\
\hline $\mathrm{C} 3-\mathrm{C} 10-\mathrm{H} 10 \mathrm{~A}$ & $110.7(12)$ & $\mathrm{C} 16-\mathrm{C} 14-\mathrm{C} 13$ & $124.27(19)$ \\
\hline $\mathrm{C} 3-\mathrm{C} 10-\mathrm{H} 10 \mathrm{~B}$ & $109.6(13)$ & $\mathrm{C} 16-\mathrm{C} 17-\mathrm{C} 18$ & $120.8(2)$ \\
\hline $\mathrm{C} 3-\mathrm{C} 10-\mathrm{H} 10 \mathrm{C}$ & $111.3(14)$ & $\mathrm{C} 16-\mathrm{C} 17-\mathrm{H} 17$ & 119.6 \\
\hline $\mathrm{C} 4-\mathrm{C} 3-\mathrm{C} 10$ & $120.92(19)$ & $\mathrm{C} 17-\mathrm{C} 16-\mathrm{C} 14$ & $121.0(2)$ \\
\hline $\mathrm{C} 4-\mathrm{C} 6-\mathrm{H} 6$ & 119.5 & $\mathrm{C} 17-\mathrm{C} 16-\mathrm{H} 16$ & 119.5 \\
\hline $\mathrm{C} 5-\mathrm{C} 4-\mathrm{C} 3$ & $118.42(17)$ & $\mathrm{C} 17-\mathrm{C} 18-\mathrm{H} 18$ & 119.9 \\
\hline $\mathrm{C} 5-\mathrm{C} 9-\mathrm{H} 9$ & 119.6 & $\mathrm{C} 18-\mathrm{C} 17-\mathrm{H} 17$ & 119.6 \\
\hline $\mathrm{C} 6-\mathrm{C} 4-\mathrm{C} 5$ & $118.45(17)$ & $\mathrm{C} 18-\mathrm{C} 19-\mathrm{C} 15$ & $120.2(2)$ \\
\hline $\mathrm{C} 6-\mathrm{C} 4-\mathrm{C} 3$ & $123.13(16)$ & $\mathrm{C} 18-\mathrm{C} 19-\mathrm{H} 19$ & 119.9 \\
\hline $\mathrm{C} 6-\mathrm{C} 7-\mathrm{C} 8$ & $120.43(19)$ & $\mathrm{C} 19-\mathrm{C} 15-\mathrm{C} 14$ & $119.95(17)$ \\
\hline $\mathrm{C} 6-\mathrm{C} 7-\mathrm{H} 7$ & 119.8 & $\mathrm{C} 19-\mathrm{C} 18-\mathrm{C} 17$ & $120.3(2)$ \\
\hline $\mathrm{C} 7-\mathrm{C} 6-\mathrm{C} 4$ & $120.92(18)$ & $\mathrm{C} 19-\mathrm{C} 18-\mathrm{H} 18$ & 119.9 \\
\hline $\mathrm{C} 7-\mathrm{C} 6-\mathrm{H} 6$ & 119.5 & $\mathrm{H} 10 \mathrm{~A}-\mathrm{C} 10-\mathrm{H} 10 \mathrm{~B}$ & $108.5(18)$ \\
\hline $\mathrm{C} 7-\mathrm{C} 8-\mathrm{H} 8$ & 119.9 & $\mathrm{H} 10 \mathrm{C}-\mathrm{C} 10-\mathrm{H} 10 \mathrm{~A}$ & $109.4(17)$ \\
\hline $\mathrm{C} 8-\mathrm{C} 7-\mathrm{H} 7$ & 119.8 & $\mathrm{H} 10 \mathrm{C}-\mathrm{C} 10-\mathrm{H} 10 \mathrm{~B}$ & $107(2)$ \\
\hline $\mathrm{C} 8-\mathrm{C} 9-\mathrm{C} 5$ & $120.81(18)$ & $\mathrm{H} 20 \mathrm{~A}-\mathrm{C} 20-\mathrm{H} 20 \mathrm{~B}$ & $109.3(18)$ \\
\hline $\mathrm{C} 8-\mathrm{C} 9-\mathrm{H} 9$ & 119.6 & $\mathrm{H} 20 \mathrm{~A}-\mathrm{C} 20-\mathrm{H} 20 \mathrm{C}$ & $108.4(19)$ \\
\hline $\mathrm{C} 9-\mathrm{C} 5-\mathrm{C} 4$ & $119.28(17)$ & $\mathrm{H} 20 \mathrm{~B}-\mathrm{C} 20-\mathrm{H} 20 \mathrm{C}$ & $104.8(17)$ \\
\hline $\mathrm{C} 9-\mathrm{C} 8-\mathrm{C} 7$ & $120.11(18)$ & & \\
\hline
\end{tabular}




$\mathrm{O} 1-\mathrm{C} 11-\mathrm{C} 12-\mathrm{C} 13$
$\mathrm{~N} 1-\mathrm{C} 5-\mathrm{C} 4-\mathrm{C} 6$
$\mathrm{~N} 1-\mathrm{C} 5-\mathrm{C} 4-\mathrm{C} 3$
$\mathrm{~N} 1-\mathrm{C} 5-\mathrm{C} 9-\mathrm{C} 8$
$\mathrm{~N} 2-\mathrm{C} 15-\mathrm{C} 19-\mathrm{C} 18$
$\mathrm{C} 1-\mathrm{O} 1-\mathrm{C} 11-\mathrm{N} 2$
$\mathrm{C} 1-\mathrm{O} 1-\mathrm{C} 11-\mathrm{C} 12$
$\mathrm{C} 1-\mathrm{N} 1-\mathrm{C} 5-\mathrm{C} 9$
$\mathrm{C} 1-\mathrm{N} 1-\mathrm{C} 5-\mathrm{C} 4$
$\mathrm{C} 1-\mathrm{C} 2-\mathrm{C} 3-\mathrm{C} 4$
$\mathrm{C} 1-\mathrm{C} 2-\mathrm{C} 3-\mathrm{C} 10$
$\mathrm{C} 3-\mathrm{C} 2-\mathrm{C} 1-\mathrm{N} 1$
$\mathrm{C} 3-\mathrm{C} 2-\mathrm{C} 1-\mathrm{O} 1$
$\mathrm{C} 3-\mathrm{C} 4-\mathrm{C} 6-\mathrm{C} 7$
$\mathrm{C} 4-\mathrm{C} 5-\mathrm{C} 9-\mathrm{C} 8$
$\mathrm{C} 4-\mathrm{C} 6-\mathrm{C} 7-\mathrm{C} 8$
$\mathrm{C} 5-\mathrm{C} 4-\mathrm{C} 3-\mathrm{C} 10$
$\mathrm{C} 5-\mathrm{N} 1-\mathrm{C} 1-\mathrm{O} 1$
$\mathrm{C} 5-\mathrm{N} 1-\mathrm{C} 1-\mathrm{C} 2$
$\mathrm{C} 5-\mathrm{C} 4-\mathrm{C} 3-\mathrm{C} 2$
$\mathrm{C} 5-\mathrm{C} 4-\mathrm{C} 6-\mathrm{C} 7$
$\mathrm{C} 5-\mathrm{C} 9-\mathrm{C} 8-\mathrm{C} 7$
$\mathrm{C} 6-\mathrm{C} 4-\mathrm{C} 3-\mathrm{C} 2$
$\mathrm{C} 6-\mathrm{C} 4-\mathrm{C} 3-\mathrm{C} 10$
$\mathrm{C} 9-\mathrm{C} 5-\mathrm{C} 4-\mathrm{C} 6$

$-174.70(15)$
$178.55(15)$
$-0.9(3)$
$-178.88(17)$
$-179.87(19)$
$83.6(2)$
$-100.8(2)$
$179.85(15)$
$0.9(3)$
$0.6(3)$
$-177.41(18)$
$-0.7(3)$
$179.64(16)$
$179.46(16)$
$0.1(3)$
$0.6(3)$
$178.14(17)$
$179.57(16)$
$-0.1(3)$
$0.1(3)$
$0.0(3)$
$0.5(3)$
$-179.31(16)$
$-1.3(3)$
$-0.4(3)$

$\mathrm{C} 9-\mathrm{C} 5-\mathrm{C} 4-\mathrm{C} 3$

$-179.86(14)$

$\mathrm{C} 9-\mathrm{C} 8-\mathrm{C} 7-\mathrm{C} 6$

$\mathrm{C} 11-\mathrm{O} 1-\mathrm{C} 1-\mathrm{N} 1$

$-0.9(3)$

17.5 (3)

$\mathrm{C} 11-\mathrm{O} 1-\mathrm{C} 1-\mathrm{C} 2$

$-162.77(16)$

$\mathrm{C} 11-\mathrm{N} 2-\mathrm{C} 15-\mathrm{C} 19$

$-179.60(17)$

C11-N2-C15-C14

C13-C14-C15-C19

$-0.5(3)$

$178.68(16)$

$\mathrm{C} 13-\mathrm{C} 14-\mathrm{C} 15-\mathrm{N} 2$

$-0.4(3)$

-179.87 (19)

$-1.2(3)$

$1.0(3)$

$1.4(3)$

$0.6(3)$

$175.70(14)$

$1.2(2)$

$-178.48(19)$

$0.1(3)$

0.4 (3)

$179.68(16)$

$-1.3(3)$

$-178.83(17)$

$1.4(3)$

$-1.6(4)$

$178.5(2)$ 